-Clinical Research.

\title{
Radiotherapy for and prognosis of breast cancer patients with local-regional recurrence after mastectomy
}

\author{
Jia-Yi Chen, ${ }^{1,2}$ Xue-Jun Ma, ${ }^{1,2}$ Wei-Bing Zhou, ${ }^{1,2}$ Yan Feng ${ }^{1,2}$ and Guo-Liang Jiang ${ }^{1,2}$
}

\author{
1. Department of \\ Radiation Oncology, \\ Cancer Hospital, \\ Fudan University, \\ Shanghai, 200032, \\ P. R. China \\ 2. Department of Oncology, \\ Shanghai Medical College, \\ Fudan University, \\ Shanghai, 200032, \\ P. R. China
}

Correspondence to: Guo-Liang Jiang

Tel. : 86.21.64175590.1407

Fax : 86.21.64174774

Email : jianggl@21cn.com

Grant : Foundation from Health

Bureau of Shanghai City

(No. 054018)

The original Chinese version of this paper is published in:Ai Zheng (Chinese Journal of Cancer) 28(10);http://www.cjcsysu.cn/cn/ article. $\operatorname{asp}$ ? id=15359)

This paper was translated into English from its original publication in Chinese.

Translated by: Chun-Yan Chen and Wei Liu

Submitted : 2008-08-25

Revised : 2009-07-12
[Abstract] Background and Objective: Controversies remain regarding the therapeutic principle for and prognosis of breast cancer patients with isolated local-regional recurrence after mastectomy. This study was to evaluate the role of radiotherapy in treating these patients and to investigate the prognosis. Methods: Clinical data of 255 breast cancer patients with chest-wall and/or regional lymph node recurrence as first failure after mastectomy from 1990 to 2005 were analyzed. All patients received radiotherapy for recurrence. Results: The median follow-up time was 45 months ( 9 months- 15.5 years). The median disease-free interval (DFI) was 22 months (2-260 months); it was 37 months in patients with positive hormonal receptor and 17 months in those with unknown or negative receptor. The 2-, 5-, and 8-year overall survival (OS) rates were $86.4 \%, 56.5 \%$, and $35.0 \%$, respectively. The median survival time was 79 months. The 2-, 5- and, 8-year local control rates were $6.1 \%, 36.3 \%$, and $27.6 \%$, respectively. Univariate prognostic analysis showed that DFI, site and number of recurrence, receptor status, short-term therapeutic response, initial $\mathrm{T}$ status and axillary involvement significantly affected the survival (all $\mathrm{P}<$ 0.05); multivariate analysis showed that DFI, receptor status, site and number of recurrence were independent prognostic factors. Prognostic index was established to classify the patients. The 2-, 5- and 8-year OS rates were $100 \%, 91.6 \%$, and $56.4 \%$ in the favorable prognosis group, $88.1 \%, 59.1 \%$, and $36.8 \%$ in the medium prognosis group, $68.0 \%, 8.5 \%$, and 0 in the poor prognosis group $(P<0.001)$. Conclusion: Radiotherapy is effective for breast cancer patients with isolated local-regional recurrence after mastectomy. Prognostic index could be applied to predict the prognosis.

Key words: breast neoplasm, chest-wall recurrence, regional lymph node recurrence, radiotherapy

Depending on clinical stage at initial treatment and prognostic factors, the rate of recurrence at the chest wall and regional lymph nodes of breast cancer patients is $5 \%-30 \%$ after modified radical mastectomy or radical mastectomy, and around $2 / 3$ of these patients showed no distant metastasis at the time of recurrence, which is called isolated loco-regional recurrence., ${ }^{1,2}$ The emergence of isolated recurrence is always an important turning point in the clinical course of breast cancer, indicating first relapse and distant metastasis in many situations. The aims of treatment on breast cancer are to effectively control the progression of local disease and reduce the occurrence of subsequent distant metastasis. The treatment principles are local resection and radiotherapy for resectable recurrent lesions, and radiation alone for 
unresectable lesions. Whether systemic therapy, including endocrine therapy and systemic chemotherapy, could improve survival of breast cancer patients with loco -regional recurrence remains uncertain. This study aimed to explore reasonable comprehensive treatment, including surgery, radiotherapy, endocrine therapy and chemotherapy, by retrospectively analyzing the clinical data of breast cancer patients with chest wall and/or regional lymph node recurrence as first failure after operation and analyze the prognostic factors.

\section{Materials and Methods}

Inclusive criteria and general data. Patients with breast cancer treated in Fudan University Cancer Hospital from February 1990 to September 2005 were selected according to criteria as follows: (1) female patients with primary breast cancer received modified radical mastectomy or radical mastectomy; (2) chest wall and/or ipsilateral regional lymph node recurrence were the first failure site and distant metastasis was excluded by imaging examination; (3) recurrent lesions were confirmed by histology or cytology; (4) the patients received radiotherapy; (5) clinical and follow-up data were intact. A total of 255 patients were eligible. The median age at diagnosis of relapse was 49 (26-79). Hormone receptor was positive in 122 patients, negative in 100 patients, and unknown in 33 patients.

Treatment of primary tumor. Of the 255 patients, 147 underwent radical mastectomy, 100 underwent modified radical mastectomy, and 8 underwent extensive radical mastectomy; 57 received postoperative radiotherapy, and the radiation fields included the supraclavicular and inner mammary lymph drainage district in 45 cases, the chest wall, supraclavicular and inner mammary district in 11 cases, and the chest wall and supraclavicular fossa in 1 case; 221 patients received adjuvant chemotherapy, 151 of them received anthracyclinefree chemotherapy and 70 received anthracyclinebased chemotherapy.

Situation and treatment of recurrent lesions. Disease -free interval (DFI) from operation to recurrence was 2-260 months, with a median of 22 months; 225 (88.2\%) patients had recurrence occurred within 5 years after operation. Of the 255 patients, $109(42.7 \%)$ had recurrence in the chest wall alone, $114(44.7 \%)$ in regional lymph nodes, 32
$(12.5 \%)$ in both the chest wall and lymph drainage district. A total of 304 recurrent lesions were found in the 255 patients, with the chest wall (141 lesions) and supraclavicular lymph nodes (99 lesioins) as the most often involved sites, another 41 recurrent lesions were found in the axillary and 23 in the inner mamma. Fifty -five patients received resection or biopsy first, 40 of them had single lesion with a diameter of $<3 \mathrm{~cm}$ and had no gross tumor residue after operation. The radiation fields of 227 patients only included recurrent lesions, and those of 29 patients were extended to cover recurrence-free areas. Among the patients with chest wall recurrence, 73 had irradiation fields only covered recurrent lesions and $1-2 \mathrm{~cm}$ surrounding normal tissue, and the rest 68 (including 2 who had received postoperative chest wall radiation) had irradiation fields covered the whole chest wall. Recurrent sites and radiation fields are detailed in Table 1.

Techniques of small field radiation on the chest wall were mainly electron beam vertical anterior field at a dose of 6-12 MeV, depending on thickness of the chest wall measured by chest CT or B ultrasound, with equivalent tissue packing of 0.5-1 $\mathrm{cm}$ that was used to increase surface dosage. Three patients were treated byhigh dose ${ }^{192} \mathrm{Ir}$ brachytherapy. Supraclavicular and inner mammary district were irradiated by ${ }^{60} \mathrm{Co}$ with single anterior filed or by mixed $6 \mathrm{MV} X$ ray and electron beam. Subaxillary fossa was irradiated by ${ }^{60} \mathrm{Co}$ or $6 \mathrm{MV} X$ ray in anterior -posterior fields with boost in accessory electronic small field. Axillary supraclavicular anterior field radiation to 40 Gy by 20 fractions was boosted by post -axillary field radiation when both sites were irradiated simultaneously. Tumor dose of electron beam was calculated by $85 \%$ of equilibrium dose. The median radiation dose was 60 Gy (42-74 Gy). Of the 190 patients who received systemic therapy, 180 received chemotherapy, 69 received endocrine therapy, and 44 received chemotherapy and endocrine therapy successively.

Follow-up and statistical analysis. The duration of follow-up was calculated from the diagnosis of recurrence. The patients were followed up for 9 months -15.5 years, with a median of 45 months, by mails and clinical visits. Re-recurrence in primary sites was defined as new recurrent lesions in the site that had primary recurrence (not always the 
Table 1 Radiation fields of the 255 breast cancer patients with local-regional recurrence

\begin{tabular}{lccccc}
\hline \multirow{2}{*}{ Site of recurrence } & \multirow{2}{*}{ Cases } & \multicolumn{4}{c}{ Radiation field (cases) } \\
\cline { 3 - 6 } & & Localized chest wall & Total chest wall & Recurrent regional nodes & Total chest wall plus regional nodes \\
\hline Chest wall & 109 & 57 & 39 & 0 & 13 \\
Regional nodes & 114 & 0 & 0 & 105 & 9 \\
Both sites & 32 & 16 & 0 & 9 & 7 \\
Total & 255 & 74 & 39 & 122 & 29 \\
\hline
\end{tabular}

area of first radiation). Re-recurrence in other sites was defined as new recurrent lesions in the affected chest wall or regional lymph nodes which were not involved in primary recurrence. Re-recurrence in primary sites and other sites, and uncontrolled primary recurrence were all defined as local failure.

SPSS11.0 software was used for data processing. Survival rate was calculated by KaplanMeier methods. The survival rates between subgroups were compared by log-rank test, and the proportions were compared by $X^{2}$ test. A $P<$ 0.05 was considered significant. Risk factors related to survival was analyzed by Cox multivariate proportion hazard model. The 95\% confidence interval $(\mathrm{Cl})$ was chose.

Establishment of prognostic index $(\mathrm{PI})$. Factors with $P<0.05$ in Cox proportion hazard model and its corresponding coefficient of variation $(\beta)$ were substituted into the following formula: $\mathrm{PI}=$ grouping value of prognosis factor $-1 \times \beta 1+$ grouping value of prognosis factor $-2 \times \beta 2+\cdots \cdots$

\section{Results}

Relationship between hormone receptor status and DFI. The median DFI of patients with positive hormone receptor was 31 months and $41.8 \%$ of the recurrence occurred within 2 years; whereas the median DFI of patients with negative hormone receptor was 17 months and $72.7 \%$ of the recurrence occurred within 2 years.

Survival rate. The median survival time of the whole group after diagnosis of recurrence was 79 months (95\% Cl: 62.5-95.8 months). The 2-, 5-, and 8 -year overall survival rates were $86.4 \%, 56.5 \%$, and $35.0 \%$; the 2-, 5-, and 8-year distant metastasis-free survival rates were $82.0 \%, 32.9 \%$, and $14.3 \%$, respectively. A total of 163 (63.9\%) patients had distant metastasis during follow-up, the median time from recurrence to distant metastasis was 15 months and the median survival time after metastasis was 49 months. The 2-, 5-, and 8-year survival rates of the 92 patients without metastasis were $96.3 \%$, 94.5\% and $94.5 \%$, respectively; while those of the 163 patients with distant metastasis were $81.5 \%, 40.8 \%$ and $15.8 \%$, respectively.

Local control and recurrence. The 2-, 5-, and 8 -year local control rates of the whole group were $56.1 \%, 36.3 \%$ and $27.6 \%$, respectively. A total of 135 (52.9\%) patients had re-recurrence during followup, including 79 had re-recurrence in primary sites, 65 had re-recurrence in other sites, and 15 patients had re-recurrence in primary sites and other sites simultaneously or successively. The median time to re-recurrence was 13 months. The chest wall was the most commonly involved site of re-recurrence, followed by the supraclavicular fossa. Of the 141 patients with primary recurrence in the chest wall, 66 had re-recurrence in the chest wall; of the 114 patients with primary recurrence in other sites, 34 had re-recurrence in the chest wall.

Univariate prognostic analysis on overall survival. Characteristics and treatment of recurrent lesions. Univariate analysis was carried out on 6 clinical factors (recurrence site, DFI, maximal diameter and number of recurrent lesions, age at recurrence and hormone receptor status), 5 therapeutic factors (resection of recurrent lesions, systemic chemotherapy or endocrine therapy for recurrent lesions, dose of radiotherapy, radiation field and short-term therapeutic response of recurrent lesions), and re-recurrence in other sites. The patients with recurrence in either the chest wall or regional lymph nodes had better prognosis that those with recurrence in both sites. The patients with longer DFI had better prognosis that those with shorter DFI. The patients with single recurrent lesion in the chest wall had better prognosis that those with multiple or diffused lesions. Hormone receptor-positive and unknown patients had better prognosis than negative patients. The patients with short-term complete remission (CR) of recurrent lesions had better 
prognosis than those with recurrence residue. The differences in survival rates were significant $(P<$ 0.05) (Table 2). The patients who had undergone resection of recurrent lesions had better prognosis than those had no resection, but without significant

Table 2 Impacts of the characteristics, treatment and outcome of recurrence on prognosis of the 255 breast cancer patients

\begin{tabular}{|c|c|c|c|c|}
\hline \multirow{2}{*}{ Parameter } & \multirow{2}{*}{ Cases } & \multicolumn{2}{|c|}{ Survival rate $(\%)$} & \multirow{2}{*}{$P$ value } \\
\hline & & 2-year & 5 -year & \\
\hline \multicolumn{5}{|l|}{ Site of recurrence } \\
\hline Chest-wall & 109 & 93.9 & 66.4 & \multirow{3}{*}{$<0.001$} \\
\hline Regional nodes & 114 & 83.6 & 55.2 & \\
\hline Chest-wall and regional nodes & 32 & 71.4 & 28.5 & \\
\hline \multicolumn{5}{|l|}{ Disease-free interval } \\
\hline$\leqslant 1$ year & 73 & 69.0 & 17.4 & \multirow{3}{*}{$<0.001$} \\
\hline$>1$ year, $\leqslant 2$ years & 68 & 88.4 & 59.5 & \\
\hline$>2$ years & 114 & 96.2 & 78.8 & \\
\hline \multicolumn{5}{|l|}{ Maximal size of recurrence } \\
\hline$\leqslant 3 \mathrm{~cm}$ & 197 & 86.1 & 56.2 & \multirow{2}{*}{0.587} \\
\hline$>3 \mathrm{~cm}$ & 58 & 87.4 & 57.7 & \\
\hline \multicolumn{5}{|l|}{ Number of recurrence } \\
\hline Single nodule & 129 & 91.5 & 70.1 & \multirow{3}{*}{$<0.001$} \\
\hline Multiple nodules & 87 & 81.3 & 48.6 & \\
\hline Diffused & 39 & 81.1 & 30.9 & \\
\hline \multicolumn{5}{|l|}{ Age at recurrence } \\
\hline$\leqslant 50$ years & 136 & 84.2 & 49.8 & \multirow{2}{*}{0.140} \\
\hline$>50$ years & 119 & 89.0 & 64.7 & \\
\hline \multicolumn{5}{|l|}{ Hormonal receptor } \\
\hline Positive & 122 & 91.4 & 70.1 & \multirow{3}{*}{$<0.001$} \\
\hline Unknown & 33 & 93.9 & 65.9 & \\
\hline Negative & 100 & 76.6 & 30.5 & \\
\hline \multicolumn{5}{|l|}{ Surgical excision } \\
\hline Yes & 55 & 96.2 & 65.8 & \multirow{2}{*}{0.051} \\
\hline No & 200 & 83.7 & 54.0 & \\
\hline \multicolumn{5}{|l|}{ Chemotherapy } \\
\hline Yes & 180 & 83.7 & 54.9 & \multirow{2}{*}{0.066} \\
\hline No & 75 & 92.9 & 59.3 & \\
\hline \multicolumn{5}{|l|}{ Endocrine therapy } \\
\hline Yes & 69 & 88.0 & 58.7 & \multirow{2}{*}{0.859} \\
\hline No & 186 & 85.5 & 56.0 & \\
\hline \multicolumn{5}{|l|}{ Radiation dose } \\
\hline$<50 \mathrm{~Gy}$ & 15 & 85.7 & 57.9 & \multirow{3}{*}{0.937} \\
\hline $50-60 \mathrm{~Gy}$ & 142 & 86.8 & 56.2 & \\
\hline$>60 \mathrm{~Gy}$ & 98 & 85.9 & 55.9 & \\
\hline Radiation field & & & & \\
\hline Confined to the recurrent region & 227 & 87.1 & 56.9 & 0.300 \\
\hline $\begin{array}{l}\text { Elective treatment to the } \\
\text { non-recurrent region }\end{array}$ & 28 & 78.8 & 65.7 & 0.390 \\
\hline Short-term response & & & & \\
\hline Complete remission & 166 & 88.2 & 63.1 & 0000 \\
\hline Partial remission & 89 & 83.4 & 45.0 & 0.009 \\
\hline Second recurrence to other regions & & & & \\
\hline No & 191 & 83.4 & 56.4 & 0696 \\
\hline Yes & 64 & 95.1 & 55.8 & \\
\hline
\end{tabular}

Table 3 Impacts of the characteristics of primary tumor and initial treatment on prognosis of the 255 breast cancer patients

\begin{tabular}{|c|c|c|c|c|}
\hline \multirow{2}{*}{ Parameter } & \multirow{2}{*}{ Cases } & \multicolumn{2}{|c|}{ Survival rate $(\%)$} & \multirow{2}{*}{$P$ value } \\
\hline & & 2-year & 5 -year & \\
\hline \multicolumn{5}{|l|}{ Primary T stage } \\
\hline $\mathrm{T} 1-\mathrm{T} 2$ & 163 & 86.5 & 68.1 & \multirow{3}{*}{0.001} \\
\hline $\mathrm{T} 3-\mathrm{T} 4$ & 50 & 76.8 & 39.2 & \\
\hline Unknown & 42 & 97.6 & 36.1 & \\
\hline \multicolumn{5}{|l|}{ Axillary node metastases } \\
\hline 0 & 66 & 100 & 84.8 & \multirow{4}{*}{$<0.001$} \\
\hline $1-3$ & 129 & 80 & 47.0 & \\
\hline$\geqslant 4$ & 33 & 81.9 & 30.3 & \\
\hline Unknown & 27 & 92.3 & 69.9 & \\
\hline \multicolumn{5}{|l|}{ Adjuvant radiotherapy and field } \\
\hline No. & 198 & 86.7 & 56.6 & \multirow{3}{*}{0.102} \\
\hline Regional nodes only & 45 & 88.4 & 64.4 & \\
\hline Regional nodes and chest wall & 12 & 73.3 & 19.6 & \\
\hline \multicolumn{5}{|l|}{ Adjuvant chemotherapy and regimens } \\
\hline No. & 34 & 85.2 & 51.9 & \multirow{3}{*}{0.126} \\
\hline Without anthracyclines & 151 & 87.4 & 60.0 & \\
\hline With anthracyclines & 70 & 84.6 & 49.2 & \\
\hline
\end{tabular}

difference in survival rate $(P=0.051) \quad$ (Table 2). Among the 114 patients with recurrence in regional lymph nodes, 81 got the supraclavicular fossa involved, and their prognosis was poorer than that of those without supraclavicular fossa involved, with 2-, 5 - and 8-year survival rates of $83.7 \%, 45.7 \%$, and $27.5 \%$ for the former, and $83.0 \%, 79.2 \%$, and $66 \%$ for the latter $(P=0.037)$.

Stage and primary treatment of primary tumor. The patients with stage T1-T2 tumor had better prognosis than those with stage T3-T4 tumor $(P=$ $0.001)$; the patients without axillary lymph node metastasis had better prognosis than those with metastasis $(P<0.001)$. No meaningful influence of adjuvant chemotherapy and radiotherapy was found on survival (Table 3).

Multivariate prognostic analysis on overall survival. COX multivariate analysis was carried out on recurrence site, DFI, maximal diameter and number of recurrent lesions, hormone receptor, surgical resection, chemotherapy and endocrine therapy, clinical CR of recurrent lesions, re-recurrence in other sites, primary $\mathrm{T}$ stage and axillary lymph node metastasis. DFI, site and number of recurrent lesions, hormone receptor status were independent prognostic factors on survival (Table 4).

Establishment of prognostic index model. DFI, site and number of recurrent lesions, hormone 
Table 4 Positive results of multivariate survival analysis and prognostic indexes

\begin{tabular}{llcccc}
\hline \multicolumn{1}{c}{ Parameter } & \multicolumn{1}{c}{ Prognostic index $^{\mathrm{a}}$} & $\beta$ & SE & Wald & $P$ \\
\hline Disease-free interval & $>2 \mathrm{yrs} />1 \mathrm{yr}, \leqslant 2 \mathrm{yrs} / \leqslant 1 \mathrm{yr}$ & 0.764 & 0.136 & 31.360 & $<0.001$ \\
Number of recurrence & Single/multiple/diffused & 0.467 & 0.144 & 10.460 & 0.001 \\
Site of recurrence & Chest wall/regional nodes/both sites & 0.318 & 0.135 & 5.546 & 0.002 \\
Hormonal receptor & Positive / unknown/negative & 0.327 & 0.127 & 6.647 & 0.001 \\
\hline
\end{tabular}

The scores for the prognostic indexes from left to right are 1,2 and 3, respectively.

receptor status were classified to three groups according to their prognostic values of scores 1-3 (Table 4). Prognostic index model: $\mathrm{Pl}=$ grouping score of DFI $\times \beta 1(0.76)+$ grouping score of recurrence site $\times \beta 2(0.32)+$ grouping score of number of recurrent lesions $\times \beta 3(0.47)+$ grouping score of hormone receptor status $\times \beta 4 \quad$ (0.33). The patients with $\mathrm{PI}$ of $<2.4$ were classified to favorable prognosis group $(3.3-0.98, \mathrm{PI})$, those with $\mathrm{PI}$ of $>$ 4.3 to poor prognosis group, and those with $\mathrm{PI}$ of 2.4-4.3 to moderate prognosis group. The 2-, 5-, and 8 -year OS rates were $100 \%, 91.6 \%$, and $56.4 \%$ in favorable prognosis group, $88.1 \%, 59.1 \%$, and $36.8 \%$ in moderate prognosis group, and $68.0 \%$, $8.5 \%, 0$ in poor prognosis group (Fig. 2), with significant difference $(P<0.001)$.

\section{Discussion}

Comparing with treatment guideline of early stage invasive breast cancer, treatment of locoregional recurrent breast cancer awaits mature consensus. Various controversies exist on predicting prognosis and selecting local and systemic treatment of recurrent breast cancer, which is still an important challenge to clinical physicians.

It is reported that most cases of the loco regional recurrence of breast cancer happened within 5 years after operation, with a peak in about the second year, and the recurrence was influenced by primary clinical stage and histological characteristics. 3 Similar to the reported results, ${ }^{3}$ the median recurrence time of our patients was 22 months after operation. The patients with negative hormone receptor had short DFI and concentrated recurrence peak, while those with positive hormone receptor had long DFI and flat recurrence time distribution. Flow cytometry revealed that the proportion of cells at phase $S$ was high in receptor -negative tumors, ${ }^{4}$ indicating that the proliferation capacity of receptorpositive tumors was relatively low, hence presented

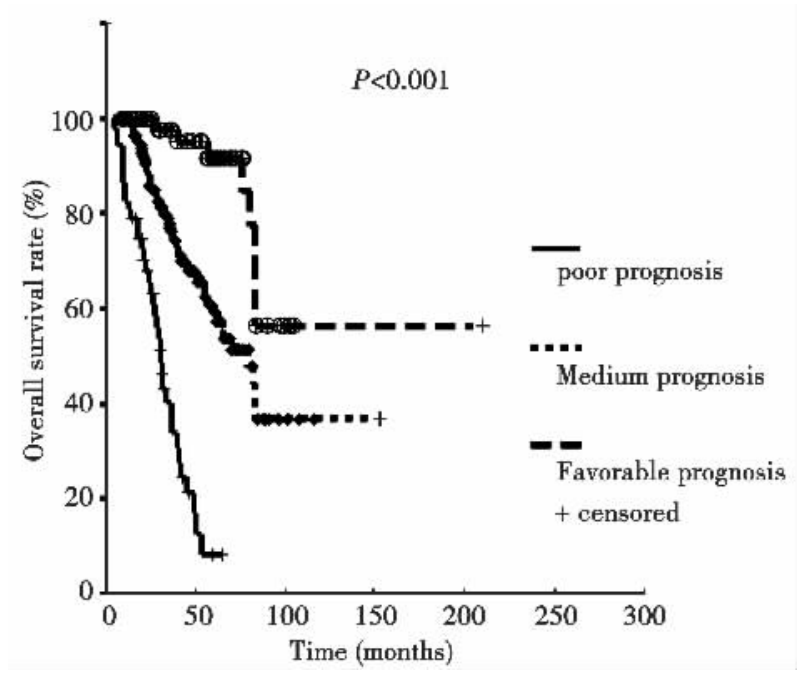

Fig. 1 Survival curves of the breast cancer patients in different prognosis groups

long DFI. Our data had approved this point. In our study, the 255 patients had 304 recurrent lesions; the order of involved frequency from high to low was the chest wall, supraclavicular fossa, axilla and inner mammary lymph nodes, and the recurrence in the chest wall and supraclavicular fossa accounted to $78.9 \%$, which was similar to those reported in literature, indicating that the chest wall and supraclavicular fossa had the highest recurrence rate and should be the major target volume of adjuvant radiotherapy after operation. 5,6

In our study, the 5-year OS rate of the whole group was $56.5 \%$ with a median survival time of 79 months, which was better than $42 \%-49 \%$ reported in most of the other papers. ${ }^{7,8}$ The possible reason lays in that more patients in our study were at early stage: $63.9 \%$ of the patients were at stages $\mathrm{T} 1-\mathrm{T} 2$, and $76.5 \%$ of the patients had no or $<3$ metastatic axillary lymph nodes. Although it is believed that recurrent breast cancer was incurable and $63.9 \%$ of the patients in our study had distant metastasis during follow-up, about $50 \%$ of the patients could survived for more than 5 years as confirmed in our 
study and other studies, ${ }^{7,8}$ and the survival situation varied significantly between subgroups. Prognostic factors affecting survival may come from characteristics and treatment responses of recurrent lesions, and also may come from stage and histological characteristics of primary tumor. Analysis on clinical characteristics of recurrent lesions showed that the span of DFI reflected the proliferation activity of tumor cells, and the number and extent of recurrent lesions reflected the tumor burthen at the emergence of recurrence. Schmoor et al. ${ }^{9}$ found that the involvement of axillary lymph nodes at primary treatment, histological grade of tumor, hormone receptor status of primary tumor and DFI were independent prognostic factors. Willner et al. $^{7}$ reported that $\mathrm{T}$ stage of primary tumor, the presence of necrosis, number and sites of recurrent lesions, the age of $>50$ years at recurrence, local control after treatment were also independent prognostic factors. $T$ stage and the status of axillary lymph nodes were both of significance in our univariate analysis, whereas no significance was found in multivariate analysis, possibly because of incomplete records of some patients. Similar to the results in most reports, ${ }^{10,11}$ no influence of primary treatment modality on prognosis was found in our data.

Integrating all prognostic factors affecting survival, subgroup of the patients with favorable prognosis could be selected out. His et al. ${ }^{10}$ found that in patients with DFI of $>2$ years, diameter of single chest wall recurrent lesion of $<3 \mathrm{~cm}$, or receiving resection, the 10 -year survival rate was up to $72 \%$. Willner et al. $^{7}$ reported that in patients with single recurrence in the chest wall or axilla, DFI of $>$ 1 year, primary tumor at stage T1-2N0, no tumor necrosis, and age of $>50$ years, the 5- and 10-year survival rates were $100 \%$ and $69 \%$. Prognostic indexes established using clinical parameters could not only practically predict the treatment outcome of a certain patient but also help to design reasonable prospective clinical trials according to various prognostic subgroups. Because of different prediction values of positive parameters, we used independent prognostic factors from COX regression analysis together with corresponding coefficient of variation ( $\beta$ value) to establish prognostic index model and defined favorable, moderate and poor prognosis subgroups. Kaplan-Meier survival test confirmed that the differences in prognoses between the subgroups were significant, indicating that this model could be used in clinic. Owing to relatively fussy calculation of $\beta$ value, to benefit practical application, the patients with 1 score for all prognostic factors, or those with DFI of $>2$ years and 2 scores for only one of the other three prognostic factors were classified to favorable subgroup.

Controversies exist regarding the value of systemic chemotherapy in treating patients with locoregional recurrence. ${ }^{11}$ In a prospective non randomized cohort study, Haylock et al. ${ }^{12}$ compared radiotherapy and chemoradiotherapy in 120 patients with recurrence who had not received adjuvant chemotherapy, and found that the risk of death in radiotherapy group was 1.39 times as much as that in chemoradiotherapy group, but without significance. In hormone receptor-positive patients, tamoxifen could reduce subsequent recurrence significantly and prolong disease-free survival as confirmed by a prospective randomized trial. ${ }^{13}$ In our study, only $36.8 \%$ of the patients with positive or unknown receptor received endocrine therapy for recurrence, and this might explain why endocrine therapy did not improve the prognosis. Kuo et al. ${ }^{14}$ reported that the patients receiving active local and systemic treatment achieved a 5 -year survival rate of $63 \%$, which indicated the potential value of systemic treatment.

Another main challenge of treatment for loco regional recurrent breast cancer is to improve local control rate and reduce subsequent recurrence. The 5 -year local control rate was $40 \%-60 \%$ as reported in literature, $7,10,15$ while it was only $36.3 \%$ in our study, and this may owing to that the 74 patients with chest wall recurrence only received local small field irradiation which led to significantly declined local control rate. Regarding the high re-recurrence rate and the chest wall being the most frequently involved site of re-recurrence, ${ }^{16}$ we suggest that not only intact local radiation to recurrent lesions but also radiation to the chest wall and supraclavicular fossa without recurrence should be given in patients with recurrence.

In our retrospective study, 52\% of the patients with chest wall recurrence received small field irradiation, and only $11 \%$ of the patients received extended field radiation to uninvolved sites. Because the main meaning of radiation fields to prognosis lays in local control rate other than survival, it may not influence the extrapolated value of the prognosis factors in survival in this study. However, the prognostic value of primary stage should not be 
ignored, and we suggest to take it in consideration in clinical practice.

In short, the breast cancer patients with locoregional recurrence have various prognoses after modified radical mastectomy or radical mastectomy. Our results indicated that DFI of $\geqslant 2$ years, single chest wall recurrent lesion and positive receptor were favorite prognostic factors. The patients with favorite prognostic factors are of radical curative value, and need timely endocrine therapy and intact loco regional radiotherapy. Meanwhile, the patients with $\mathrm{DFI}$ of $<1$ year, diffused recurrent lesions and negative receptor have poor prognosis, with a 5-year survival rate of $<10 \%$, and need salvage systemic therapy as main treatment and radiotherapy as palliative treatment. The patients with moderate prognosis have a 5 -year survival rate of $>50 \%$, and need loco -regional radiotherapy and systemic therapy, especially endocrine therapy for patients with positive hormone receptor. These patients are suitable subjects for prospective clinical trials of combined chemoradiotherapy.

\section{References}

[1] Katz A, Strom EA, Buchholz TA, et al. Locoregional recurrence patterns after mastectomy and doxorubicin-based chemotherapy: implications for postoperative irradiation $[\mathrm{J}]$. J Clin Oncol, 2000,18(15) :2817-2827.

[2] Yang MT, Rong TH, Huang ZF, et al. Clinical analysis of resectable breast cancer, a report of 6263 cases [J]. Ai Zheng, 2005,24(3):327-331. [in Chinese]

[3] Pisansky TM, Ingle JN, Schaid DJ, et al. Patterns of tumor relapse following mastectomy and adjuvant systemic therapy in patients with axillary lymph node-positive breast cancer. Impact on clinical, histopathologic and flow cytometric factors $[\mathrm{J}]$. Cancer, 1993 ,72(4) : 1247-1260

[4] Wenger CR, Clark GM. S-phase fraction and breast cancer, a decade of experience $[\mathrm{J}]$. Breast Cancer Res Treat, 1998,51 (3) : 255-265

[5] Wang SL, Li YX, Yu ZH. Targetting in postmastectomy radiotherapy for early stage breast cancer [J]. Chin J Radiat Oncol, 2001,10(4):223-227. [in Chinese]
[6] Pierce LJ. The use of radiotherapy after mastectomy: a review of the literature [J]. J Clin Oncol, 2005,23(8):1706-1717.

[7] Willner J, Kiricuta IC, Kolbl O. Locoregional recurrence of breast cancer following mastectomy: Always a fatal event? results of univariate and multivariate analysis $[\mathrm{J}]$. Int $\mathrm{J}$ Radiat Oncol Biol Phys, 1997,7(4):853-863.

[8] Nielsen HM, Overgaard M, Grau C, et al. Loco-regional recurrence after mastectomy in high-risk breast cancer-risk and prognosis. An analysis of patients from the DBCG 82b\&c randomized trials $[\mathrm{J}]$. Radiother Oncol, 2006,79 (2) : 147 155.

[9] Schmoor C, Sauerbrei W, Bastert G, et al. Role of isolated locoregional recurrence of breast cancer, Results of four prospective studies [J]. J Clin Oncol, 2000,18(8) : 1696-1708.

[10] Hsi RA, Antell A, Schultz DJ, et al. Radiation therapy for chest wall recurrence of breast cancer after mastectomy in a favorable subgroup of patients $[\mathrm{J}]$. Int $\mathrm{J}$ Radiat Oncol Biol Phys, 1998,42(3):495-499.

[11] Recht A, Come SE, Troyan SL. Local-regional recurrence after mastectomy or breast-conserving therapy $[\mathrm{M}]$. Disease of the breast. Second ed. Harris JR, ed. Philadelphia: Lippincott Wiliams \& Wilkins, 2000 :731-749.

[12] Haylock BJ, Coppin CM, Jackson J, et al. Localregional first recurrence after mastectomy: prospective cohort studies with and without immediate chemotherapy $[\mathrm{J}]$. Int $\mathrm{J}$ Radiat Oncol Biol Phys, 2000,46(2):355-362.

[13] Borner M, Bacchi M, Goldhirsch A, et al. First isolated locoregional recurrence following mastectomy for breast cancer: results of a phase III multicenter study comparing systemic treatment with observation after excision and radiation $[\mathrm{J}]$. $\mathrm{J}$ Clin Oncol, 1994,12(10):2071-2077.

[14] Kuo SH, Huang CS, Kuo WH, et al. Comprehensive locoregional treatment and systemic therapy for postmastectomy isolated locoregional recurrence $[\mathrm{J}]$. Int $\mathrm{J}$ Radiat Oncol Biol Phys, 2008, 72 (5) : 1456-1464.

[15] Halverson KJ, Perez CA, Kuske RR, et al. Locoregional recurrence of breast cancer: a retrospective comparison of irradiation alone versus irradiation and systemic therapy $[\mathrm{J}]$. Am J Clin Oncol, 1992,15(2):93-101.

[16] Chen JY, Guo XM, Zhang Z, et al. Factors influencing local control after radiotherapy of isolated local-regional recurrence following mastectomy [J]. Chin J Radiat Oncol, 2008,17(1):1116. [in Chinese] 